\title{
Stress, Cortison und Homöostase
}

\section{Künstliche Nebennierenrindenhormone und physiologisches Gleichgewicht, 1936-1960}

\section{Lea Haller}

\begin{abstract}
Stress, Cortisone and Homeostasis. Adrenal Cortex Hormones and Physiological Equilibrium, 1936-1960

This article investigates the emergence of the concept of stress in the 1930s and outlines its changing disciplinary and conceptual frames up until 1960. Originally stress was a physiological concept applied to the hormonal regulation of the body under stressful conditions. Correlated closely with chemical research into corticosteroids for more than a decade, the stress concept finally became a topic in cognitive psychology. One reason for this shift of the concept to another discipline was the fact that the hormones previously linked to the stress concept were successfully transferred from laboratory to medical practice and adopted by disciplines such as rheumatology and dermatology. Thus the stress concept was dissociated from its hormonal context and became a handy formula that allowed postindustrial society to conceive of stress as a matter of individual concern. From a physiological phenomenon stress turned into an object of psychological discourse and individual coping strategies.
\end{abstract}

Keywords: Stress, general adaptation syndrome, hormones of the adrenal cortex, cortisone therapy, coping

Schlüsse/wörter: Stress, Allgemeines Adaptationssyndrom, Nebennierenrindenhormone, Cortisontherapie, Stressbewältigung

"No concept in the modern psychological, sociological, or psychiatric literature is more extensively studied than stress." Stevan E. Hobfoll, der in den 1980er Jahren Stressbewältigung als sozialstrukturabhängiges Ressourcenmanagement formulierte, beließ es nicht bei dieser Diagnose. „I submit that stress is also the socialpsychological concept of greatest interest to Western society. Perhaps no other psychosocial topic is discussed more often in the lay 
press or kaffeeklatsches." (Hobfoll 1998: 1) Man kann ihm keine Betriebsblindheit unterstellen. Stress hat in der zweiten Hälfte des 20. Jahrhunderts als Feld wissenschaftlicher Auseinandersetzung und - zeitlich ein wenig verschoben - als Alltagsbegriff und Selbstbeschreibungskategorie der westlichen Gesellschaft eine enorme Dynamik entwickelt. In der Medizin- und Wissenschaftsgeschichte erfährt diese Konjunktur zunehmende Aufmerksamkeit. Verschiedene Autoren haben zur Historisierung des chamäleonartigen Begriffs beigetragen und seine hohe Wandlungs- und Anschlussfähigkeit thematisiert. ${ }^{1}$ Stress migrierte von der experimentellen Hormonforschung über die Militärpsychiatrie und verschiedene medizinische Spezialgebiete in die Alltagssprache der postmodernen Gesellschaft, wo er anscheinend wie kein anderer Topos geeignet war, die Grenzen der Anpassungsfähigkeit an permanent sich verändernde, nicht antizipierbare Belastungen zu beschreiben. Damit kann er, wie Cornelius Borck feststellte, gleichsam als symptomatisch für das letzte Drittel des 20. Jahrhunderts gelesen werden: als „Pathologie der Flexibilisierung" (Borck 2004: 83).

Ich blende in diesem Artikel zurück auf die Entstehungsbedingungen des biologischen Stresskonzepts. Im Zentrum des Interesses stehen die reziproken Dynamiken zwischen der physiologisch-biologischen Theorie und der chemisch-pharmazeutischen Hormonforschung in den 1930er und 1940er Jahren. Die globalen Konzepte zur Steuerung und Regulation von Körperfunktionen, in deren Kontext der Stressbegriff entstand, sollen hier an die lokalen Bedingungen industrieller Forschung und Entwicklung geknüpft werden. Die chemisch-pharmazeutischen Fallbeispiele sind der Forschung und Entwicklung bei der Schweizer Pharmafirma Ciba entnommen (bis 1945 offiziell „Gesellschaft für Chemische Industrie Basel"). Ich stütze mich dabei auf Archivrecherchen im Firmenarchiv Novartis Basel und den Nachlass des Chemikers Tadeus Reichstein im Staatsarchiv Basel-Stadt. Ciba arbeitete auf dem Gebiet der Corticosteroide eng mit Reichstein zusammen und konnte 1938 ein erstes künstliches Nebennierenrindenhormon in die klinische Prüfung geben. Die Diskussion der physiologischen Modelle basiert auf publizierten Quellen, wobei sich in den Archivbeständen direkte Verbindungen zwischen der chemischpharmazeutischen Entwicklung und der physiologischen Forschung nachweisen lassen. ${ }^{2}$ Reichstein hat in Absprache mit seinen Vertragsfirmen wiederholt Substanzen an Hans Selye, einen jungen Endokrinologen und Biochemiker aus Montreal, geschickt. Selye, der sich für die Funktion dieser Hormone interessierte, hat sie für seine Studien an Modellorganismen verwendet. 
1936 publizierte Selye in der Zeitschrift Nature einen Artikel mit dem Titel „A Syndrome Produced by Diverse Nocuous Agents". Er beschrieb darin seine Beobachtung, dass Versuchstiere auf schädliche Einwirkungen aller Art - Unterkühlung, operativer Eingriff, übermäßige Muskelbelastung oder Vergiftung - immer ähnlich reagieren. Diese Antwort des Körpers auf ,damage as such” (für die er später den physikalischen Begriff „Stress” benutzte) sei prinzipiell auf Anpassung ausgerichtet. Der Organismus versuche, sich in einem stereotyp ablaufenden Prozess auf die veränderten Bedingungen einzustellen und trotz anhaltender Belastung wieder normal zu funktionieren. Da dieser Prozess unabhängig von der Art der schädlichen Ursache ablaufe, schlage er dafür den Namen „General Adaptation Syndrome” vor (Selye 1936).

Verantwortlich für diese physiologische Anpassungsreaktion seien, so Selye, die Hormone der Nebennierenrinde, mit deren Strukturaufklärung sich um 1936 verschiedene Chemiker beschäftigten - allerdings nicht, um aus dem Nebennierenrindenextrakt eine „Adaptationssubstanz” zu isolieren, sondern um jene Substanz zu finden, die am effizientesten die tödlichen Mangelerscheinungen bei Nebennierenunterfunktion beheben könnte. 1939 brachte die Ciba eine erste synthetische Rindensubstanz auf den Markt („Percorten”). Bekannt wurden die Nebennierenrindenhormone allerdings erst zehn Jahre später, als unter dem Handelsnamen „Cortison” ein von Tadeus Reichstein und Edward C. Kendall bereits 1936 isoliertes Hormon (Reichsteins „Substanz Fa”; Kendalls „Compound E”) auf den Markt kam. 1950 erhielten Reichstein und Kendall zusammen mit dem Rheumatologen Philip Hench für ihre Forschungen über Nebennierenrindenhormone den Nobelpreis für Medizin und Physiologie. Im gleichen Jahr publizierte Selye seine erste Monographie zum Stress und legte damit den Grundstein für eine begriffliche Karriere weit über das biologische Labor hinaus.

Zwischen 1936 und 1950 tat sich ein Raum der physiologischen, biologisch-endokrinologischen, chemischen, pharmazeutischen und medizinischen Forschungspraxis auf, deren Resultate in den 1950er Jahren zur breiten Anwendung von künstlichen Nebennierenrindenhormonen einerseits und zu einem adaptierfähigen Stressbegriff andererseits führten. Diese doppelte Genese von Stress und Corticosteroiden, ihre parallele Karriere in den 1950er Jahren und schließlich ihre Trennung in zwei disparate Bereiche sind Gegenstand der folgenden Überlegungen. Dass der Stress ab 1950 und vor allem ab Mitte der 1960er Jahre zu einer nachgerade ubiquitären Formel wurde, hängt - so die Vermutung - eng mit der breiten klinischen Anwendung von Corticosteroiden in den 1950er 
Jahren zusammen. Die an Wunder grenzenden Möglichkeiten der medizinischen Cortisontherapie bedingten gleichzeitig die Popularität des Stresskonzepts und seine Entkopplung vom endokrinologischen Kontext. Die Dissoziation von Stress und Hormonen bot neuen Lesarten und Aufladungen Raum. In den 1950er Jahren und vor allem ab Mitte der 1960er Jahre wurde der Stress - zunächst im Rahmen der Militärpsychiatrie - für Psychologen und Psychiater interessant. Für sie waren die Hormone der Nebennierenrinde nicht mehr von Bedeutung, wohl aber der semantische Rahmen der Steuerung und Regulation von Körperfunktionen, der eng mit dem biologischen Stresskonzept verbunden war. Er wurde in einer Zeit des sozialen und strukturellen Wandels von der Biologie auf die Lebenswelt übertragen.

\section{Ein erstes synthetisches Hormon}

Die Nebenniere ist ein lebenswichtiges Organ. 1855 hatte der englische Arzt Thomas Addison eine kleine Schrift publiziert (Addison 1855), in der er darlegte, dass eine tödlich endende Krankheit mit Symptomen wie Bronzefärbung der Haut, Übelkeit und Schwäche bis zur totalen Asthenie ursächlich mit der Zerstörung der Nebenniere zusammenhing; die Krankheit wurde später nach ihm „Addisonsche Krankheit” genannt. In den ersten beiden Dekaden des 20. Jahrhunderts entwickelte sich unter Physiologen eine Auseinandersetzung um die Frage, was genau nun lebenswichtig sei: das Hormon des Nebennierenmarks, Adrenalin, das bereits 1904 synthetisch hergestellt werden konnte und im Handel erhältlich war, oder die Nebennierenrinde, deren chemische Zusammensetzung man nicht kannte. Als es um 1927 gelang, aus Nebennierenrinden adrenalinfreie Extrakte herzustellen, und damit die Lebenszeit von adrenalektomierten Tieren verlängert werden konnte (Swingle 1927, Hartman/MacArthur/ Hartman 1927), war eine langjährige Streitfrage entschieden und das Feld für eine medizinische Therapie der Addisonschen Krankheit mit tierischen Extrakten geöffnet.

Die Lösung des physiologischen Problems wurde Anfang der 1930er Jahre als chemisch-pharmazeutisches Problem reformuliert. Verschiedene Pharmafirmen begannen sich für das Nebennierenrindenextrakt "Cortin” zu interessieren. ${ }^{3}$ Sie wurden zum Drehpunkt einer Logistik von Schlachtabfällen, Organextrakten und isolierten reinen Substanzen, die in Tierversuchen wiederum auf ihre Wirksamkeit getestet werden mussten. ${ }^{4}$ An der Eidgenössischen 
Technischen Hochschule (ETH) Zürich beschäftigte sich ab 1934 der Chemiker Tadeus Reichstein mit dem Nebennierenrindenextrakt. ${ }^{5}$ Er bezog es von der holländischen Pharmafirma Organon, die auf Organextrakte spezialisiert war - die Firma war 1923 von einem Direktor von Schlachtbetrieben gegründet worden. Als Reichstein 1936 plausibel machen konnte, dass die Substanzen aus der Nebennierenrinde zur Klasse der Steroide gehören, geriet er in Konflikt mit Leopold Ruzicka, dem Vorsteher des Instituts für organische Chemie der ETH. Ruzicka arbeitete selber auf dem Gebiet der Sexualhormone - ebenfalls Steroidhormone - und hatte mit der Ciba einen Vertrag unterzeichnet, der ihr im Gegenzug für die massive finanzielle Unterstützung des Instituts die alleinige Nutzung seiner Forschungsergebnisse sicherte (Ratmoko 2008). Es bestand nun akut Gefahr, dass Wissen über den Aufbau von Steroidhormonen via Reichstein an die ausländische Konkurrenz ging. Umgekehrt kamen Reichstein und Organon nicht umhin, die Ciba mit an Bord zu holen, weil sie auf dem Steroidgebiet wichtige Patente besaß. 1936 nahmen Ciba, Organon und Haco (ein Nahrungsmittelkonzern, der Reichsteins Forschungen mitfinanzierte) Verhandlungen auf, um die Nutzungsrechte auf dem Cortingebiet zu regeln und 1937 unterzeichneten die Parteien einen entsprechenden Vertrag. ${ }^{6}$

Organon lieferte weiterhin das Rohmaterial und prüfte in ihren biologischen Laboratorien die von Reichstein aus Nebennierenrindenextrakt isolierten Substanzen auf ihre Wirksamkeit. Anfang der 1930er Jahre hatte man in verschiedenen Laboratorien Verfahren entwickelt, um die biologische Aktivität von Nebennierenrindensubstanzen zu testen. Während in den USA vor allem der sogenannte Hundetest angewendet wurde (Swingle/Pfiffner/ Vars 1934), prüfte Organon nach „Everse-de Fremery” an Ratten (Everse/de Fremery 1932). Man entfernte den Tieren die Nebennieren, substituierte sie mit Nebennierenrindenextrakt, bis sie sich erholt hatten, und injizierte darauf in regelmäßigen Abständen die zu prüfende Substanz. Dabei wurde die Dosis so lange verringert, bis erste Zeichen von Nebennierenrindeninsuffizienz auftraten. Die Dosis, die diese gerade noch verhinderte, nannte man eine ,dogunit” beziehungsweise eine „Ratten-Einheit”. Sie galt als Vergleichsgröße, um die Wirksamkeit verschiedener Substanzen zu messen (Bomskov/Bahnsen 1935: 1).

Die Evaluation von reinen Hormonen hing damit von einer physiologischen Interpretationspraxis ab, mit der an bestimmten Zeichen des Mangels abgelesen wurde, welche der isolierten Substanzen am ehesten die Ausfallserscheinungen beheben könnte. 
Waren nebennierenlose Tiere nach der Injektion einer Substanz aus Nebennierenrinden über längere Zeit lebensfähig, so hatte die Substanz den „Überlebenstest” bestanden und konnte als aktiv eingestuft werden. Bis 1941 waren 28 verschiedene Substanzen aus Nebennierenrindenextrakt isoliert worden, von denen sechs im Überlebenstest die gewünschte Wirkung zeigten: Sie konnten Tiere ohne Nebennierenrinde am Leben erhalten. ${ }^{7}$ Eine von Reichstein und seiner Assistentin Marguerite Steiger 1937 teilsynthetisch hergestellte Substanz (Reichstein/Steiger 1937) erwies sich dabei als am stärksten wirksam: die „Substanz Y” (Desoxycorticosteron, kurz Doc beziehungsweise Doca für das pharmazeutisch verwendete Desoxycorticosteron-Acetat). Nach klinischen Prüfungen brachte die Ciba sie 1938 unter dem Handelsnamen Percorten als erstes synthetisches Nebennierenrindenpräparat auf den Markt. ${ }^{8}$

Die Addisonsche Krankheit war die einzige sichere Indikation für Percorten. Sie war aber ein relativ seltenes Phänomen, und das Interesse der Ciba richtete sich noch vor der Markteinführung ihres neuen Hormonpräparats auf potentielle weitere Anwendungsfelder. Um die klinische Prüfung in erfolgversprechende Richtungen zu lenken, verfolgte man in der Propagandaabteilung aufmerksam die physiologische Forschung auf dem Gebiet der Nebennierenrinden. In der umfangreichen Bibliothek des Unternehmens wurden die Publikationen von Selye und anderen Physiologen auf mögliche Indikationen für das neue Präparat hin studiert. Besondere Aufmerksamkeit schenkte man dabei jenen Schädigungen, die gemäß Selye das General Adaptation Syndrome und damit die körpereigene Ausschüttung von Nebennierenrindenhormonen auslösten. Im September 1939 erklärte sich der Leiter des Schweizer Militärkrankenhauses bereit, Percorten bei der Behandlung von postoperativem Schock, bei Verbrennungen und nach schweren Infektionskrankheiten zu prüfen. ${ }^{9}$ Im Sommer 1940 wurde auf einer Sitzung des Pharmazeutischen Komitees bei der Ciba protokolliert: „Percorten und Perandren als Gegengifte bei Nieren- und Leberschädigungen: Auf Grund neuer Arbeiten und einzelner gemeldeter Fälle soll versucht werden, die beiden Präparate in diese Richtung zu lenken (siehe Selye, J. of Pharmacology and exp. Therap. April 1940)." ${ }^{\prime 0}$ Indem man bei der Ciba Selyes Allgemeines Adaptationssyndrom als Reaktion auf konkrete Schädigungen des Organismus interpretierte, konnte die medizinische Anwendung von Percorten auf eine Reihe spezifischer Indikationen ausgedehnt werden. Insbesondere die Anwendung bei postoperativem Schock und bei Schwangerschaftstoxikosen wurde in den 1940er Jahren zur Routine. 


\section{„Addisonismus” und die Weisheit des Körpers}

Mit der Erfindung einer neuen Krankheit wurde dem Percorten ein weiteres Anwendungsgebiet erschlossen. Man begann sich bei der Ciba für Erschöpfungszustände zu interessieren, die zwar kein „echter Addison” waren, die aber bestimmte Symptome in abgeschwächter Form aufwiesen. Das Augenmerk richtete sich auf Zustände verminderter Nebennierenrindenfunktion, oder wie es in einer Sitzung des Pharmazeutischen Komitees hieß: „Wichtig wird die Feststellung von chronischen Rindeninsuffizienzen sein, welche nicht bis zu einem sichtbaren Addison führen (Adynamie). Diese Fälle werden dem Präparat auch die breitere Anwendungsmöglichkeit geben."11 Unter dem Namen „relative Nebenniereninsuffizienz" oder „Addisonismus" fand die chronische Rindeninsuffizienz in den 1940er Jahren Eingang in die Therapie (Abderhalden 1946: 117).

Auf einer internen Tagung der Ciba am 1. Oktober 1948, auf der Mitarbeiter der Propagandaabteilung geschult wurden, hielt ein Vertrauensarzt der Ciba ein Referat zu diesem „praktisch außerordentlich wichtigen Anwendungsgebiet des Percorten”. ${ }^{12}$ Als Symptome der relativen Nebenniereninsuffizienz nannte er ,allgemeines Schwächegefühl, rasche Ermüdbarkeit, evtl. Appetitlosigkeit und Gewichtsabnahme". Ziemlich charakteristisch sei „die sog. ,Abendadynamie', d. h. die Patienten, sofern es sich um Leute handelt, die ihrer Berufsarbeit nachgehen, fühlen sich gegen den Abend zu besonders erschöpft und aufgebraucht" (ebd.). Zum Nachweis der relativen Nebenniereninsuffizienz existiere eine Reihe klinischer Funktionsproben: Belastungsproben des Elektrolyt- und Wasserhaushalts, des Kohlehydratstoffwechsels und des Zirkulationsapparates. Sie könnten allerdings nur in der Klinik durchgeführt werden, weshalb sie für den Praktiker nicht von großem Interesse seien. Der Praktiker verfüge jedoch über ein einfaches und zuverlässiges Hilfsmittel zur Diagnose, ,und zwar über den ,therapeutischen Test' mit Percorten" (ebd.). Liege aufgrund der Anamnese ein Verdacht auf relative Nebennierenrindeninsuffizienz nahe, so empfehle es sich, eine Behandlung mit Percorten durchzuführen. Besserten sich die Beschwerden wie Adynamie, Appetitlosigkeit und Schwindelgefühl, so spreche dies für die Richtigkeit der Diagnose.

In den 1940er Jahren war es der Ciba also gelungen, einerseits das unspezifische Adaptationssyndrom Selyes auf konkrete Schockzustände, Verbrennungen, Leber- und Nierenschädigungen zu reduzieren, andererseits die handfeste, tödlich verlaufende Addi- 
sonsche Krankheit auf einen unspezifischen Übermüdungszustand auszudehnen. Die Therapie der „relativen Nebenniereninsuffizienz" ging mit einer Umkehrung der Kausalordnung einher: Nicht mehr die klinisch diagnostizierte Krankheit führte zur Verschreibung eines Medikaments, sondern der Wirkstoff wurde zu diagnostischen Zwecken eingesetzt. ${ }^{13}$ Und nicht mehr die Feststellung der Zerstörung der Nebennierenrinde (und damit die Addisonsche Krankheit) stand beim Addisonismus im Zentrum, sondern die Frage, wo eine Dysfunktion der Nebennierenrinde überhaupt beginne und ein Einsatz von Percorten bereits gerechtfertigt sei. Mit dem Einsatz von Percorten zur Diagnose und Therapie des Addisonismus wurde die medizinische Intervention auf einen pathological range, das heisst einen dehnbaren pathologischen Bereich zwischen absoluter Gesundheit und absoluter Krankheit, ausgeweitet. Diese dynamische Interpretation eines Krankheitsbildes hatte ein Analogon in den 1930er Jahren: Künstliches Vitamin $\mathrm{C}$ wurde bei der eigens dafür geschaffenen Indikation „Avitaminose" verabreicht, da Skorbut als einzige medizinische Indikation klinisch irrelevant war (Bächi 2009).

Die Therapie des Addisonismus basierte auf der Vorstellung eines physischen Gleichgewichts, das unter bestimmten Umständen gestört wird und das per Hormongabe wiederhergestellt werden kann. Diese Vorstellung stand in der Tradition einer physiologischen Theorie, die sich seit der Jahrhundertwende mit der Regulation im Organismus auseinandersetzte. Sie erhielt in den 1930er Jahren vor allem durch Walter B. Cannon Auftrieb. ${ }^{14}$ Cannon war Professor in Harvard und seit 1914 Präsident der American Physiological Society. Sein 1932 veröffentlichtes Standardwerk The Wisdom of the Body markierte den Höhepunkt einer Forschungstradition, die sich vom minutiösen Studium einzelner Organe entfernt hatte und das holistische Zusammenspiel aller Körperfunktionen ins Zentrum rückte. Als Ernest Starling 1905 für aktive physiologische Substanzen den Begriff Hormone prägte, hatte er sich den Organismus nicht mehr als mechanisches Ensemble von funktional ausdifferenzierten Zellen - Virchows „Elementarorganismen" - vorgestellt, sondern als intelligentes, chemisch gesteuertes Adaptationssystem. ${ }^{15}$ Von einer Rede Starlings (Starling 1923) borgte sich Cannon den Titel zu seinem Buch. The Wisdom of the Body war in der Überzeugung geschrieben, dass der Organismus zu intelligenter Anpassung fähig sei und auf äußere Veränderungen mit einer inneren Justierung antworte. Für die koordinierten physiologischen Prozesse, die den stabilen (aber nicht statischen) Zustand des Organismus gewährleisten, 
schlug Cannon den Begriff Homöostase vor: „The word does not imply something set and immobile, a stagnation. It means a condition which may vary, but which is relatively constant." (Cannon 1932: 24)

Cannons Überlegungen zur Regulation und zur Homöostase basierten weitgehend auf seinen eigenen Untersuchungen zur Wirkungsweise des Adrenalins. In einem 1914 veröffentlichten Artikel referierte er seine Beobachtung, dass sich die Adrenalinausschüttung unter Schmerz oder bei Gefühlen wie Angst und Wut auf den Blutdruck, die Durchblutung der wichtigsten Organe, die Herz- und Magentätigkeit sowie auf den Blutzucker und die Muskelarbeitsleistung auswirke (Cannon 1914). Diese „emergency function” bereite den Organismus darauf vor, effizient auf eine Gefahr reagieren zu können. Während des Ersten Weltkriegs verband Cannon seine Arbeiten zum Adrenalin mit einem klinischen Syndrom: Für den traumatischen Schock von Soldaten prägte er den Begriff „Flight-or-Fight-Response” (Cannon 1915).

\section{Zwei Kontrahenten}

Für Selye waren Cannons Arbeiten zum Adrenalin, zum traumatischen Schock und zur Homöostase eine wichtige Referenz, als er 1936 seinen ersten Entwurf zum General Adaptation Syndrome publizierte. Bei der Anpassungsreaktion des Organismus auf schädliche Einflüsse unterschied er drei Stadien (Selye 1936; siehe Abb. 1). In einer ersten Phase reagiere der Organismus mit Schock (alarm reaction) und mit einer erhöhten Ausschüttung von Adrenalin. Darauf folge eine längere Phase, in der sich der Organismus auf die veränderte Lage einzustellen versuche, einen relativ stabilen Zustand erreiche und wieder normal funktioniere (stage of resistance). In dieser Phase, in der das physiologische Gleichgewicht wiederhergestellt werde, sei das Adrenalin allerdings nicht mehr von Belang, dafür würden vermehrt Nebennierenrindenhormone ausgeschüttet. Nach einer gewissen Zeit beobachte man wieder ähnliche Symptome wie in der Alarmphase, die jetzt aber mit totaler Erschöpfung und dem Verlust der Anpassungsfähigkeit einhergingen (state of exhaustion).

Die Alarmreaktion in Selyes dreistufigem Modell entspricht Cannons Flight-or-Fight-Reaktion. Selye dehnte das binäre ReizReaktionsschema Cannons jedoch auf einen nichtlinearen Verlauf aus und wertete bei der Anpassungsleistung des Organismus die 
Abb. 1 Das General Adaptation Syndrome (Selye 1946: 123)

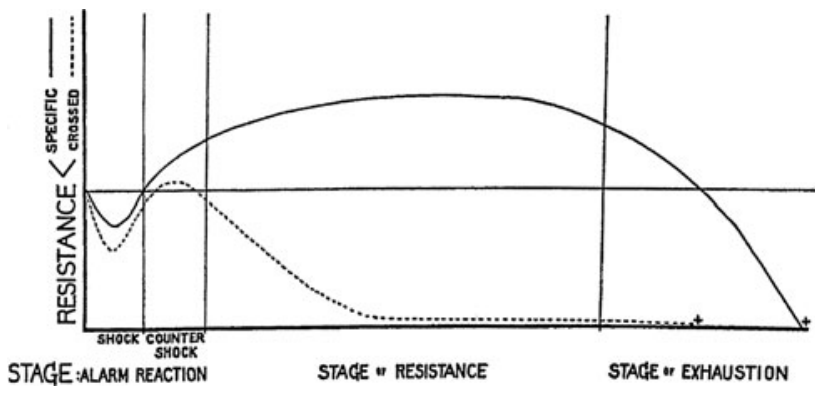

Bedeutung der Nebennierenrindenhormone höher als jene des Adrenalins. Damit wurde Cannon nicht nur zu seinem ersten Gewährsmann, sondern auch zu seinem größten Kontrahenten. Cannon interessierte sich dezidiert nur für die Funktion des Sympathikus und für die Katecholamine (Adrenalin, Noradrenalin und Dopamin), also für jene Infrastruktur, die für die schnelle Reaktion zuständig ist. Selye hingegen interessierte sich sozusagen für die longue durée der physischen Anpassungsleistung, denn: „In some cases one cannot flee." (Selye 1975: 90) Dieses Problem des physischen Umgangs mit permanenter Störung habe Cannon mit seiner Fixierung auf das Adrenalin nicht lösen können. Der langfristige Adaptationsprozess sei keine Flight-or-Fight-Reaktion, so Selye, sondern bestehe eher darin, dass die schädliche Ursache ignoriert oder unschädlich gemacht werde, ,and that can be accomplished best, as far as we know today, with steroid hormones" (ebd.: $90 \mathrm{f}$.). In der zweiten Phase des General Adaptation Syndrome gelinge es dem Organismus bei erhöhter Corticoidausschüttung zumindest für eine bestimmte Zeit, sich auf Kälte, Schock, Verletzungen, Infektionen, Vergiftungen und Entzündungen einzustellen und wieder normal zu funktionieren. Während man bei der Ciba die Vermarktung des ersten synthetischen Nebennierenrindenhormons an die Kompensation einer physischen Unterfunktion koppelte, ging es Selye um eine übergreifende Theorie des physischen Umgangs mit „Krankheit an sich”, ja um eine „unified theory of disease” (Selye 1956: 12 f.). Krankheit bedeute nicht einfach Leiden, sondern sei ein Kampf, um das homöostatische Gleichgewicht im Körper aufrecht zu erhalten.

In den 1940er Jahren wurde das selektive Interesse der Pharmaindustrie an den Forschungen Selyes jedoch zu einem prekären Verhältnis. Grund dafür waren in erster Linie neue biologische Testmethoden, mit denen nicht mehr die Totalwirkung einer einzelnen Substanz geprüft wurde, sondern ihr Effekt auf spezifische 
Stoffwechselfunktionen wie Muskelarbeitsleistung, Glukosegehalt des Blutes, Natrium- und Chlorretention oder die Resistenz gegen Schock. In einem solchen, von Dwight J. Ingle entwickelten Test war auf einmal nicht mehr Percorten (Doca) am wirksamsten (Ingle 1941). Ingle untersuchte die Muskelarbeitsleistung von adrenalektomierten Ratten unter Einfluss verschiedener Nebennierenrindenhormone, und hier zeigten die bisher vernachlässigten und äußerst schwer zu synthetisierenden Hormone von der Bauart des Compound E die größte Wirkung. Auch Selye konnte Mitte der 1940er Jahre plausibel machen, dass für die Reaktion auf Langzeitbelastungen nicht jene Nebennierenrindenhormone zuständig waren, die wie Percorten vor allem auf den Elektrolythaushalt wirkten. In seinem über hundert Seiten langen Artikel The General Adaptation Syndrome and the Diseases of Adaptation legte er 1946 dar, dass vielmehr der Kohlehydratstoffwechsel und damit die Hormone der Bauart des Compound E bei der körpereigenen Adaptation von zentraler Bedeutung seien.

\section{Kollabierende Piloten und ein Stressindex}

Nach Ausbruch des Zweiten Weltkriegs begann ein Wettlauf um die Synthetisierung der bisher vernachlässigten Hormone, die auf den Kohlehydratstoffwechsel und die Muskelleistung wirken. Besonders in den USA wurde die Suche nach einer praktikablen Synthese des Compound E zur staatlich forcierten Kriegsforschung (Rasmussen 2002a, b). Grund dafür mag ein Gerücht gewesen sein, wonach die deutsche Luftwaffe über Nebennierenrindenstoffe verfügte, die gegen Hypoxämie wirksam seien und den Piloten eine Flughöhe von über 40.000 Fuß ermöglichten (Birch 1992, Uchibayashi 2001).

Der Körper der Militärpiloten war ein Paradebeispiel für die Extreme der physischen Anpassungsfähigkeit. An ihnen exemplifizierte Selye 1946 seine Hypothese, dass jeder Mensch nur über eine bestimmte „Adaptabilität” oder „Anpassungsenergie” verfüge, die im Verlauf längerer Anpassungsleistung verbraucht werde. Piloten, so hatte er beobachtet, konnten sich zwar meist über längere Zeit an wiederholte Belastungen anpassen, kollabierten aber nach einer Zeit der scheinbaren Gewöhnung plötzlich (Selye 1946: 179). Nicht die auslösende Ursache bestimme also den Verlauf des General Adaptation Syndrome, sondern die physische Verfassung und die individuelle „Stressgeschichte” des Organismus. 
Auch Hudson Hoagland, Professor für Physiologie in Harvard, hatte zwei Jahre zuvor festgestellt, dass Instruktoren der US Air Force nach 90 Flugstunden pro Monat trotz ausgezeichneter Konstitution völlig erschöpft waren. Zusammen mit seinem Kollegen Gregory Pincus führte er eine Reihe von Untersuchungen durch, deren Resultate er in einem Artikel von 1944 mit dem visionären Titel Adventures in Biological Engineering referierte. In einem großen Bogen griff er einleitend die in der Physiologie paradigmatischen Themen des homöostatischen Gleichgewichts, der Anpassung und der hormonellen Regulation im Körper auf: „Man, together with other higher vertebrates, has developed some elegant automatic mechanisms for regulating the physical and chemical properties of his blood and body fluids." (Hoagland 1944: 63) In den letzten Jahren, mit der Entwicklung der Fliegerei, sei der Mensch allerdings dazu übergegangen, sich in einem Umfeld frei bewegen $\mathrm{zu}$ wollen, für das ihn seine Evolutionsgeschichte nicht ausgerüstet habe. Es gebe Indizien dafür, so Hoagland, dass die Hormone der Nebennierenrinde eine bedeutende Rolle spielten, wenn sich der Organismus auf eine technisch ermöglichte Lebenswelterweiterung und den damit einhergehenden Stress einzustellen versuche (Pincus/Hoagland 1943: 173).

Mit einer von Wilhelm Zimmermann entwickelten Methode, Steroidhormone (sogenannte 17-Ketosteroide) im Urin nachzuweisen, wollten Hoagland und Pincus bei ihren Untersuchungen herauszufinden, ob die plötzliche Ermüdung der Piloten von einer erhöhten Ausschüttung von Corticoiden unter Stress verursacht wurde. Angehende Piloten mussten sich in eine Art Flugsimulator setzen und einen Lichtstreifen auf einem vorgeschriebenen Pfad über einen Bildschirm bewegen, wobei jeder Fehler sofort registriert wurde. Nach drei bis vier Stunden konzentrierter Bedienung waren die Studenten erschöpft und auch nach viel Training wuchs die Fehlerrate in der zweiten Hälfte der Einsatzzeit erheblich. Hoagland und Pincus stellten fest, dass die 17-Ketosteroidausschüttung in der zweiten Runde, also wenn die Versuchsteilnehmer bereits erschöpft waren, deutlich anstieg. Den erhöhten Output unter Stress nannten sie ,stress 17-ketosteroids” (Hoagland 1944: 65). Sie hatten also eine Versuchsanlage entwickelt, um aus einem physiologischen Phänomen einen quantitativen Wert zu machen: Für Stress gab es nun einen messbaren Index.

Ziel von Hoagland und Pincus war es aber nicht nur, den Stress von Kampfpiloten zu quantifizieren. Sie suchten nach einem Steroidhormon, das den Organismus belastungsresistenter machen würde. Das auf dem Markt erhältliche Doca wirkte jedoch, wie man 
wusste, auf den Wasser-Elektrolyt-Haushalt und war, wie Hoagland annahm, wohl vor allem für den erhöhten Harndrang unter Stress verantwortlich. Die interessanteren Hormone, die auf den Blutzuckergehalt und die Muskelleistung wirkten (wie das Compound E), waren für größere experimentelle Studien nach wie vor nicht verfügbar. ${ }^{16}$ Hoagland und Pincus untersuchten den Effekt von verschiedenen Substanzen und kamen zu dem Schluss, dass ein einfaches Steroidhormon, das ihnen von Schering zur Verfügung gestellt wurde, sich bei Piloten positiv auf die Arbeitsleistung auswirkte: Pregnenolon. Es war so etwas wie die Mutter aller Corticosteroide; man konnte es relativ einfach aus Cholesterol aufbauen.

Nach erfolgversprechenden Versuchen mit Pregnenolon bei Militärpiloten weiteten Hoagland und Pincus ihre Studien auf ein Sample aus, das als repräsentativ für die soziale Vielfalt der unter Stress leidenden Bevölkerung angesehen werden konnte. Akademiker unter Leistungsdruck, müde Fabrikarbeiter und Menschen mit psychischen Störungen rückten ins Zentrum des Interesses. Ihre ersten Studien mit Arbeitern führten Hoagland und Pincus in der Farber Leather Company of Worcester durch, wo Arbeiter aus gegerbten Tierhäuten Leder für Schuhinnensohlen zuschnitten. Acht Wochen lang erhielt abwechselnd eine Gruppe Pregnenolon und die andere Gruppe ein Placebo (Pincus/Hoagland 1945: 342 f.). Im Gegensatz zu den Piloten waren hier allerdings keine statistisch signifikanten Unterschiede in der Produktivität festzustellen. Hoagland und Pincus machten den fixen Wochenlohn und die Sollvorgabe für die Anzahl zuzuschneidender Sohlen, das heißt die fehlenden Leistungsanreize des Unternehmens dafür verantwortlich. In der Parker Manufacturing Company of Worcester fanden sie Arbeiter und Arbeiterinnen, die unter höherem Leistungsdruck standen. Sie arbeiteten an Revolverdrehbänken und stellten Bajonette für Armeewaffen her, arbeiteten rund um die Uhr in drei Schichten und wurden pro Stück bezahlt. Die Fabrikkrankenschwester verteilte täglich das als „Vitamin-Pille” deklarierte Medikament, wobei jeweils eine Hälfte der Schicht Pregenolon bekam und die andere ein Placebo. Die Ergebnisse waren erstaunlich. Die Produktion der Pregnenolon-Probanden stieg in dieser Fabrik erheblich. In der zweiten Woche erhöhten auch die PlaceboArbeiter, die in der gleichen Produktionshalle unmittelbar neben den anderen arbeiteten, ihre Leistung.

Die Forscher interpretierten die Ergebnisse dahingehend, dass bei der Steroidmedikation mit Pregnenolon die Motivation der Versuchsteilnehmer eine entscheidende Rolle spiele. Die Armee- 
piloten seien hochmotiviert gewesen. Sie pflegten einen wetteifernden Truppengeist und seien überzeugt gewesen, dass die aus der Studie gewonnenen Erkenntnisse für die Fliegertruppe wichtig seien. Entsprechend gut waren die Resultate; die höhere Konzentrations- und Leistungsfähigkeit lag bei 10-30\%. Die untersuchten Studenten dagegen seien in ihrem letzten CollegeSemester gewesen und hätten 1943 darauf gewartet, jederzeit in die Armee eingezogen zu werden. „Boredom and indifference to pushing themselves was characteristic of the student group in contrast to the aviators." (Ebd.: 345) Bei den Fabrikarbeitern gab es zwei Gruppen: solche ohne Leistungsdruck, bei denen das Präparat keine Produktionssteigerung erzeugte, und solche mit Leistungsdruck, bei denen die Produktivität stieg. In einer weiteren Studie mit „mental patients” habe es wiederum keine signifikanten Veränderungen gegeben bezüglich des Erreichens bestimmter Fertigkeiten. „In this last series, motivation to make high scores was in general at a very low ebb." (Ebd.) Eine einheitliche, kontrollierbare Leistungssteigerung war von der Medikation mit Pregnenolon also nicht zu erwarten. Die Effekte des verabreichten Steroidhormons basierten vorwiegend auf der Motivation der Probanden, die sich wiederum auch mit dem Placebo günstig auf die Leistung auswirkte. Die experimentelle Stresstherapie von Hoagland und Pincus kam nie in ein Stadium klinischer Prüfung.

\section{Das „Compound E” und die Rheumatologie: eine Allianz}

In einem anderen Zusammenhang ergab sich wenig später jedoch ein folgenreiches Bündnis zwischen einem Steroidhormon und einer medizinischen Indikation. 1946 gelang Lewis Sarett, der bis 1942 eng mit Edward C. Kendall zusammengearbeitet und anschließend zum Pharmakonzern Merck gewechselt hatte, eine Teilsynthese des Compound E auf der Basis von Gallensäure (Sarett 1946). Die Synthese über 37 Stufen ergab achtzehn Milligramm des kostbaren Stoffes und war im großtechnischen Maßstab nicht durchführbar. Das Verfahren wurde mehrfach abgeändert und vereinfacht, bis 1948 eine ausreichende Menge für einen klinischen Versuch vorhanden war: hundert Milligramm (Sarett/Wallis 1947). Merck versuchte die Allokation der knappen Ressource und die Durchführung der klinischen Versuche zuerst an die National Academy of Sciences (NAS) zu delegieren und hoffte, damit an die Evaluation des Penicillin und die während des 
Zweiten Weltkriegs etablierte Kooperation zwischen Industrie, Regierung und unabhängiger Expertenkommission anschließen zu können (Marks 1992). Klinisch, institutionell und politisch hatte sich die Situation aber grundlegend geändert. Es war - vor allem angesichts der verschwindend kleinen vorhandenen Menge beim Compound E kein Konsens darüber zu erreichen, wie es am besten zu prüfen sei und was überhaupt zuerst getestet werden sollte. Nachdem sich herausgestellt hatte, dass die akademische Kommission der NAS nicht die richtige Instanz war, um zwischen den verschiedenen Akteuren zu vermitteln, entschied man sich bei Merck für eine unsystematisch durchgeführte klinische Prüfung.

Kendall informierte seinen Kollegen Philip Hench, den Leiter der Rheumaabteilung an der Mayo-Klinik, dass das verfügbare Compound E für einen klinischen Versuch reichen würde. Hench war sehr interessiert an dem neuen Hormon. Er hatte bereits in den 1930er Jahren beobachtet, dass die Schmerzen von Rheumapatienten bei Gelbsucht, Hepatitis oder während einer Schwangerschaft auf unerklärliche Weise gemildert waren, wenn auch nur vorübergehend (Hench 1938). Könnten rheumatische Erkrankungen eine Folge gescheiterter Adaptation des Körpers auf schädliche Einflüsse sein, sogenannte Adaptationskrankheiten? Selye hatte in einem 1944 publizierten Artikel diese These aufgestellt (Selye 1944) und schien zwei Jahre später kaum mehr Zweifel daran zu haben:

For instance, such conditions as nephritis, rheumatic fever or acute gastric ulcers, in some instances indubitably occurred as the result of an acute infection but in other instances they could, with an equal degree of certainty, be traced to an exposure to intense cold, an intoxication or an emotional stimulus. (Selye 1946: 190, 192)

Dass das Compound E auf irgendeine Weise ursächlich mit dem Auftreten beziehungsweise Verschwinden der entzündlichen Krankheit verknüpft sein könnte, war jedoch pure Spekulation; man wusste nichts Konkretes über die Pathogenese rheumatischer Erkrankungen. Aufgrund der therapeutischen Verlegenheit und Polypragmasie in der Rheumatologie setzte Hench trotzdem gewisse Hoffnungen in die neue Substanz. $\mathrm{Zu}$ verlieren gab es nicht viel, haftete der Rheumatologie doch seit jeher das Stigma der Quacksalberei an. Ihre Heilverfahren deckten ein Spektrum ab, das kaum etwas unversucht ließ: Behandlungen mit Proteinschock, Bienengifteinspritzungen, Fiebertherapie, Schwefelinjektionen, Gummibandagen, Streptokokken-Impfungen, Hydro-, Thermo-, Elektro-, Röntgenstrahlen- und Kurzwellentherapie, die Gabe von Salizylaten (Aspirin) sowie die durch den Franzosen Jaques Forestier 
entwickelte Therapie mit Goldsalz-Injektionen (Klinkenberg 1987: 100, Copeman 1964). Hench hatte also langjährige Erfahrung mit theoretisch nicht abgesicherter, empirischer Therapie im Trialand-Error-Modus. In einem 1938 verfassten Artikel über die positive Wirkung der Gelbsucht auf Rheumatismus hatte er bereits gefragt, ob es nicht wahrscheinlich sei, dass der Unterschied zwischen einer vorläufigen, befristeten Besserung und der endgültigen, anhaltenden Besserung, die wir Heilung nennen, bloß quantitativ sei? Neueste Erfolge bei der Behandlung bestimmter Krankheiten hätten gezeigt, dass es sich lohne, den Hinweisen der Natur zu folgen, egal wie irrational diese Hinweise seien, anstatt weiterhin Methoden anzuwenden, die zwar rational, aber nicht sehr erfolgreich seien. So habe es zum Beispiel keinen rationalen Grund gegeben, Malaria bei Neurosyphilis anzuwenden. „Originally, behind this venture was simply the unexplained fact ,that it worked'." (Hench 1938: 395)

Dass es funktioniert, war beim Compound E sofort evident. Hench hatte die Substanz einer Patientin injiziert, die seit Jahren an schwerer rheumatischer Arthritis litt und bisher auf keine Behandlung angesprochen hatte (Hench 1949). Der Effekt wird auch ein halbes Jahrhundert später noch in der Rhetorik biblischer Wunderheilung wiedergegeben: „Schon nach zwei Tagen konnte sich die Patientin im Bett bewegen, am dritten Tag hatte sie keine Schmerzen mehr und stand erstmals auf. Nach einer Woche Behandlung nahm sie ein Taxi, fuhr in die Stadt und machte drei Stunden lang Einkäufe." (Kaiser/Kley 2002: 5) Am 13. April 1949 berichteten Hench und sein Team auf der wöchentlichen Konferenz der Mayo-Klinik vor einem größeren Ärztekreis über die antirheumatische Wirkung der neuen Substanz. Bei diesem Treffen war bereits ein amerikanischer Journalist anwesend, und acht Tage später erschien auf der Titelseite der New York Times ein erster Bericht über das neue Hormon (Laurence 1949). Einen Monat später verkürzten Kendall und Hench den langen chemischen Namen des Compound E (17-hydroxy-11-dehydro-corticosterone) $\mathrm{zu}$,"cortisone”, und wenige Wochen später wurde die Substanz auf dem 7. Internationalen Kongress für Rheumakrankheiten der Fachwelt vorgestellt. ${ }^{17}$

Als nach Monaten der Knappheit größere Mengen Cortison produziert werden konnten und das Medikament für die breitere Therapie verfügbar war, konnte sich die Rheumatologie als wissenschaftliche Disziplin profilieren. Die Rheumatologen machten die korrekte Cortisonanwendung zu ihrem primären therapeutischen Programm; alternative Therapieformen traten in den Hintergrund. 
„The Cinderella of medicine had met her Prince Charming”, hieß es 1949 in einem Kongressbericht (Annals of the Rheumatic Diseases 1949: 302). Die Deutungs- und Anwendungshoheit konnte sich die Rheumatologie auch noch erhalten, als sich in den an der MayoKlinik durchgeführten weiteren klinischen Tests herausstellte, dass die Beschwerden nach Absetzen der Therapie sofort wieder auftraten und dass es zahlreiche unerwünschte Nebenwirkungen gab (Hench/ Kendall/Slocumb/Polley 1950). Damit wurde es jedoch unwahrscheinlich, dass Cortison die Ursachen rheumatischer Erkrankungen bekämpfte und dass Rheuma - wie von Selye angenommen - eine Adaptationskrankheit sei. Die Analogie zur Insulintherapie bei Diabetes erwies sich als Chimäre und der in der anfänglichen Euphorie kreierte Neologismus „rheumocrinology” (Annals of the Rheumatic Diseases 1949: 302), der Rheumatologie und Endokrinologie zu einer sinnhaften Einheit verknüpfte, wurde wieder aufgegeben. Im September 1951 hielt Kendall vor der British Medical Association ein Referat, in dem er angesichts der Unkenntnis über den Wirkungsmechanismus vor allem darauf hinwies, dass Cortison den Grundstein für weitere Forschung gelegt habe. Der vielversprechendste Aspekt der klinischen Studien sei, dass Cortison nicht nur bei Rheuma, sondern bei einer Vielzahl von Krankheiten wirke. Diese unerwartete, weitreichende Anwendungsmöglichkeit des Cortisons möge darauf zurückgehen, dass es irgendeinen all diesen Krankheiten gemeinsamen ursächlichen Faktor kontrolliere. Was das genau sein könnte, wisse man noch nicht. Fest stehe jedoch, "that the hypotheses of the adaptation syndrome of Selye were not acceptable" (Kendall 1951: 456).

\section{Der Stress und die Hormone dissoziieren: ein Ausblick}

Sowohl in der Medizin als auch in der chemischen Forschung und in der pharmazeutischen Industrie hatte das Cortison einen äußerst dynamisierenden Effekt. Während die Rheumatologen in Cortison einen Garant für Wissenschaftlichkeit sahen und verschiedene Chemiker an einfacheren Syntheseverfahren arbeiteten, erweiterte sich die Palette der Indikationen rasant - neben rheumatischen Erkrankungen wurden Erfolge bei Augen-, Lungen- und Hautkrankheiten, bei Asthma und Allergien aller Art vermeldet (Copeman 1953). Zeitgleich mit der Ausdehnung der Cortisontherapie auf verschiedene medizinische Gebiete erlangte auch Selyes Allgemeines Adaptationssyndrom breitere Aufmerksamkeit. Unter dem Begriff Stress führte Selye 1950 seine eigenen Arbeiten 
zur Adaptation, Claude Bernards Begriff des milieu intérieur, Cannons Konzept der Homöostase, Frank A. Hartmans ,general tissue hormone"-Theorie des Cortins (Hartman 1934) sowie verschiedene Beobachtungen $\mathrm{zu}$ unspezifischen therapeutischen Wirkstoffen zusammen, ,into a single unified biological system” (Selye 1950b: 1383). Was in den 1930er Jahren als unspezifisches Syndrom formuliert wurde, bekam 1950 einen holistischen kybernetischen Anspruch, den Selye rhetorisch als Chiasmus anlegte: „Anything that causes stress endangers life, unless it is met by adquate adaptive responses; conversely, anything that endangers life causes stress and adaptive responses." (Ebd.) Anpassungsfähigkeit und Stressresistenz bedingten sich gegenseitig und seien fundamentale existentielle Parameter. In einem komplexen Regulationskreis - der sogenannten Hypophyse-Nebennieren-Achse werde die physische Reaktion auf Stress gewährleistet. In einem Rückkopplungsmechanismus werde die Corticoidausschüttung vom in der Hypophyse gebildeten Adrenocorticotropen Hormon $(\mathrm{ACTH})$ kontrolliert, und dieses wiederum werde vom Corticoidgehalt im Blut reguliert. Ohne explizit auf die Kybernetik zu verweisen, konzipierte Selye den Stress in den 1950er Jahren als sich selbst steuernden Anpassungsmechanismus (siehe Abb. 2). ${ }^{18}$

In den folgenden Jahren erschienen alle großen Publikationen Selyes zum Stress, bis hin zu seinem als Autobiographie zu lesenden The Stress of Life (Selye 1950a, 1952, 1956). Die Nähe zur Kybernetik dürfte für die Popularität, die der Stress in den 1950er Jahren in Fachkreisen erhielt, ebenso förderlich gewesen sein wie sein formelhafter, ubiquitärer Charakter. Der Stressbegriff expandierte in zahlreichen neuen Wortschöpfungen für verschiedene klinische Krankheitsbilder und psychische Störungen. In Nominalkomposita wurde ihm jeweils eine Konstituente beigeordnet, die das universelle Syndrom spezifizierte: In den wissenschaftlichen Publikationen war von „cardiovascular stress”, „occupational stress”, „surgical stress”, „climacteric stress”, ,,mental stress” und ,emotional stress” die Rede.

In der Steroidforschung und der Therapie mit Corticosteroiden spielten der Stress und das General Adaptation Syndrome indessen keine bedeutende Rolle mehr. Das Konzept der sogenannten „Adaptationskrankheiten" stand im Gegenwind; nur vereinzelt wurden Dermatosen oder Allergien noch als Folgen gescheiterter Adaptation und damit als Stresskrankheiten aufgefasst (zum Beispiel Wolff/Wolf/Hare 1950). Gerade in der Rheumatologie, der Vorzeigedisziplin der Cortisontherapie, fand Selyes Vorschlag, dass die gesamte Medizin und Pharmakologie neu überprüft werden müsse, „um unspezifische von spezifischen Effekten - in Krankheit und in 


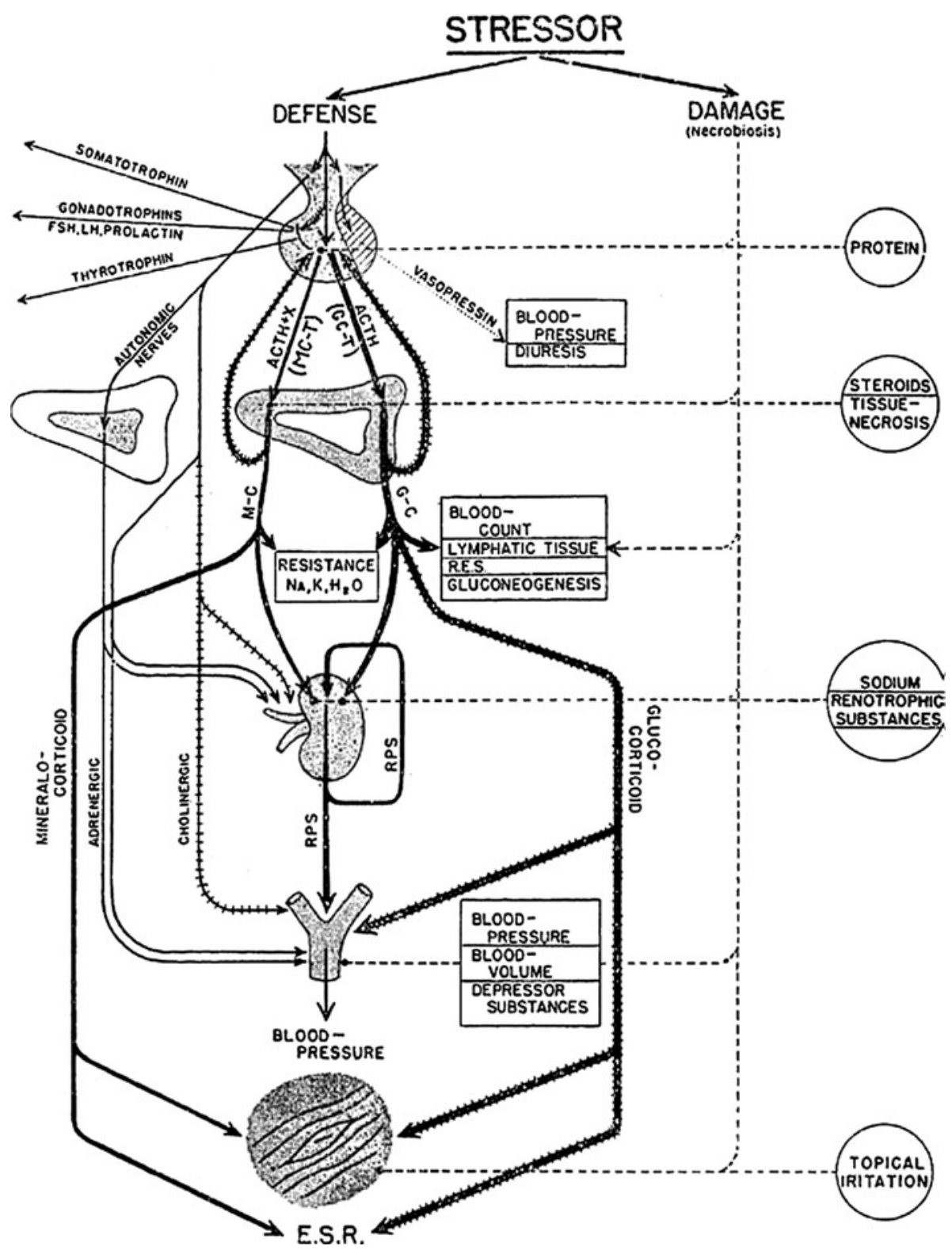

Therapie - abzugrenzen", auf sehr pragmatische Art und Weise Eingang in die Praxis: als Symptombekämpfung zahlreicher abzugrenzen" (Selye 1952: 160), Das kausale Krankheitskonzept, das mit der Bakteriologie Ende des 19. Jahrhunderts zum therapeutischen Leitprinzip geworden war (Sinding 1991, Carter 2003), wurde mit der Cortisontherapie aufgegeben zugunsten einer pragmatischen Medikalisierung, die sich ausschließlich über den Effekt recht-
Abb. 2 Das General Adaptation Syndrome (Selye 1950b: 1384) 
fertigte. Das hatte Auswirkungen auf die klinische Praxis. An die Stelle von Fragen nach der Ätiologie wurden mit dem Cortison Fragen nach der adäquaten Dosierung, der optimalen Darreichungsform und der Langzeitbetreuung chronisch kranker Patienten akut.

Während die Corticosteroidtherapie in den 1950er Jahren zur Sache einzelner medizinischer Fachgebiete wurde und der Stress als Epitheton in ganz unterschiedlichen Symptomkomplexen auftauchte, etablierte sich eine neue Lesart des Stresskonzepts. In der Militärpsychiatrie wurde der Stress nach dem Zweiten Weltkrieg - analog zum traumatischen Schock der Soldaten nach dem Ersten Weltkrieg - als typisches Syndrom der Kampftruppen diskutiert (Binneveld 1997). Im März 1953 fand an der Army Medical Service Graduate School in Washington ein Symposium zum Thema Stress statt, auf dem einerseits die einschlägigen biologisch-physiologischen Themenfelder besprochen wurden und andererseits eine behavioristische und psychologische Diskussion des Stresses ausgetragen wurde. Es war von „behavioral regulation of homeostasis" die Rede, von "chronic situations evoking psychological stress” oder ",psychological adaptive process in life-threatening injuries". ${ }^{19}$ In der psychiatrischen Diskussion in den USA der 1950er Jahre kombinierte man scheinbar mühelos biologistisch-deterministische Konzepte mit psychosozialen Verhaltensmustern. Stress bot sich in dieser Verquickung als ideales missing link zwischen manifesten physischen Schäden und der individuellen Reaktion auf Dauerbelastungen an.

Für den psychologischen Stressbegriff, der sich in den 1960er Jahren etablierte, waren die Arbeiten von Richard S. Lazarus, Professor für Psychologie in Berkeley, stilbildend. Die semantische Nähe zu Selyes Arbeiten ist frappant. Auch in der psychologischen Variante von Lazarus ging es um Stimuli und Reaktion, um Anpassung und Regulation und um die Wiederherstellung eines Gleichgewichts. Lazarus interessierte sich allerdings nicht mehr für die hormonell gesteuerte Reaktion des Organismus, die während der Evolution erworbene Anpassungsfähigkeit des Organismus. Entscheidend für die Stressreaktion sei nicht, ob ein physiologischer Adaptationsmechanismus eingeleitet werde, sondern ob etwas überhaupt als Gefahr erkannt werde. Die Bedeutung eines gefährlichen Stimulus hänge also davon ab, wie er aufgrund der psychischen Disposition eines Individuums wahrgenommen werde, kurz „on learning and on past experience" (Lazarus 1966: 393-395).

Damit wurde die individuelle Stressbewältigung zum zentralen Anliegen. Die kognitive Emotionstheorie war auf bewusstes, zielgerichtetes Handeln ausgerichtet und machte den Umgang mit 
Stress zu einer lernbaren Angelegenheit (Lefrançois 2006: 95, Schönbächler/Schulthess 2009). Für die Kontrolle und Steuerung des persönlichen Stresshaushalts benutzte Lazarus den sowohl in der Biologie als auch in der Informationstheorie etablierten Begriff der „self-regulation” (Lazarus 1977). Der Transfer des biologischphysiologischen Stresskonzepts in die Psychologie machte aus der passiven, hormongesteuerten Adaptationsleistung des Organismus einen Imperativ zur Selbstkontrolle.

\section{Der Ort des Wissens}

„Mythos, Labor und Klinik sind eng miteinander verflochten” was Donna Haraway für die Immunologie feststellte (Haraway 1995: 162), gilt für die Forschung an Nebennierenrindenhormonen und die Genese des Stressbegriffs in hohem Maß. Trägt man den unterschiedlichen Kontexten Rechnung, in denen sie erforscht wurden, wird die Dynamik des interdisziplinären Austauschs evident. Die Akteure des Wissens - Physiologen, Biologen, Chemiker, Pharmakologen - waren trotz ihrer unterschiedlichen disziplinären und institutionellen Verankerungen aufeinander angewiesen (vgl. von Schwerin 2009: 5). Das Experimentalsystem der doppelten Genealogie des Stresses und des Cortisons bestand gerade darin, dass Substanzen, physiologische Interpretationen, theoretische Konzepte und die Resultate biologischer und klinischer Prüfungen über disziplinäre Grenzen hinweg ausgetauscht wurden.

Das Labor wäre nicht der richtige Ort gewesen, um den Transfer des Adaptationssyndroms von der Biochemie in verschiedene medizinische Teildisziplinen und schließlich in den Alltag einer flexibilisierten Gesellschaft zu untersuchen. Ebensowenig war die Entwicklung der Corticosteroide Resultat eines kanalisierten Wissens- und Technologietransfers zwischen Hochschule und Industrie (Grandin/Wormbs/Widmalm 2004). Die Wechselwirkungen zwischen physiologischen Modellen, chemischer Synthese und biologischen Tests waren keine Sache unternehmerischer Planung oder staatlicher Forschungsförderung im Stil der Big Science (Nye 1996, Galison/Hevly 1992). Sie waren schlicht der Tatsache geschuldet, dass sich die Interessen der auf Steuerungsund Adaptationsprozesse spezialisierten Physiologen, der an Naturstoffen arbeitenden Chemiker und der auf Steroidsynthesen setzenden Pharmaindustrie in den Nebennierenrindenhormonen trafen und dass Probleme oder Resultate des einen Bereichs Auswirkungen auf die Forschung anderer Bereiche hatten. 
Dass der Stress in der zweiten Hälfte des 20. Jahrhunderts sowohl zu einem ausdifferenzierten Forschungsgegenstand als auch zu einem äußerst populären Begriff wurde, hat verschiedene Gründe. Einer davon ist, wie gezeigt wurde, die Entkopplung des Stresskonzepts von den Hormonen im Zuge der breiten klinischen Anwendung von Corticosteroiden in den 1950er Jahren. Die bis dahin eng mit der Corticoidforschung verknüpfte Theorie der biologischen Adaptation an schädliche Umwelteinflüsse verlor mit dem Erfolg der Cortisontherapie ihre konkrete klinische Dimension. Übrig blieb ein biologisches Konzept, das sich aufgrund seiner kybernetischen Rahmung für eine sozialpsychologische Neuformulierung anbot. Der Grund für den unglaublichen Erfolg, der dem Stress in der zweiten Hälfte des 20. Jahrhunderts beschieden war, dürfte also darin liegen, dass der Begriff als Variable funktionierte, und dass er gleichzeitig - aufgrund der Entwicklungen in der chemisch-pharmazeutischen Forschung einen soliden Referenzrahmen erhalten hatte, der entscheidend $\mathrm{zu}$ seiner Legitimation beitrug. Mit dieser ,soliden Variable” wurde ab den 1960er Jahren der Umgang mit dem stressbedingten Ausnahmezustand als individuelle Aufgabe formuliert. Aus dem hormonellen Steuerungsmechanismus, der in Selyes Konzept dem Organismus unter Stress das homöostatische Gleichgewicht garantiert hatte, wurde in der postindustriellen Gesellschaft eine Aufforderung zur Regulierung der eigenen Work-Life-Balance.

\section{Anmerkungen}

1 Viner 1999, Jackson 2006, Borck and Cornelius 2004, Hofer 2006, Kury 2008, Siegrist 2001.

2 Nachlass Reichstein, Staatsarchiv Basel-Stadt (im Folgenden StaBS), PA 979a K 11-3 1 Wissenschaftliche Korrespondenz mit Ciba 1937-1943, Brief vom 9. Januar 1942; ebda., K 11-2 2: Wissenschaftliche Korrespondenz mit Organon 1937-1938, Briefe vom 1. November 1938 und 7. November 1938; ebd., K 11-24 Wissenschaftliche Korrespondenz mit Organon Inc., 1940-1949, Brief vom 6. Oktober 1945; Ciba-Archiv, FO PH 5.01, Biologisch-chemische Besprechung Dr. Miescher-Prof. Meier, Protokoll Nr. 4 vom 17. Juni 1953.

3 Darunter Organon, Schering, Glaxo, Merck und Ciba.

4 Zum Verhältnis von Hochschul- und Industrieforschung s. Schmoch 2003, Gugerli/Kupper/Speich 2005, Bürgi/Strasser 2005; zur industriellen Hormonforschung s. Sinding 2001, Gaudillière 2004, Rasmussen 2005.

5 StaBS, PA 979a, K 1-2 6: Cortin-Journale 1-5.

6 CIBA-Archiv, C-RE/V, Recht, Verträge Nr. 386 a/b/c, HACO A.G./ Prof. Dr. T. Reichstein (und Prof. C.A. Grob), Interne Notizen.

7 StaBS, PA 979a, J 2-2: Vorträge über Cortin. J 2-2 1: Vortrag von T. Reichstein in Bern, 1941.

8 CIBA-Archiv, Vg 1.10.1, Protokoll über die Ph.C.-Sitzung Nr. 1 vom 12. Januar 1939, S. 2. 
9 Ebd., Protokoll über die Ph.C.-Sitzung Nr. 11 vom 25. September 1939, S. 1 f.

10 Ebd., Protokoll über die Ph.C.-Sitzung Nr. 12 vom 3. Juni 1940, S. 3.

11 Ebd., Protokoll über die Ph.C.-Sitzung Nr. 11 vom 25. September 1939, S. 3.

12 Ebd., PE 8.01, Pharma, Aus- und Fortbildung, Propagandisten-Tagungen 1947, 1957. Akten Prof. Dr. Rolf Meier.

13 Als Katalysator für die Percorten-Anwendung bei Addisonismus wirkte die Entwicklung einer neuen Applikationsform: Ciba hatte ein PercortenGlykosid entwickelt, das per Injektion verabreicht wurde und dessen Wirkung sofort einsetzte. CIBA-Archiv, Vg 1.10.1, Protokoll Nr. 6 über die Ph.C.-Sitzung vom 25. Mai 1943.

$14 \mathrm{Zu}$ Cannon s. Brooks/Koizumi/Pinkston 1975, Benison/Wolfe/Barger 1987, Lederer 1992, Cannon 1994, Wolfe/Barger/Benison 2000.

15 S. Sinding 1991, Stoff 2004, 2008, Sengoopta 2006, Tanner 2008. Zur Kontroverse, die die empirische Hormonforschung und Starlings spekulative Visionen innerhalb der Physiologie auslösten, s. Long Hall 1976.

161946 erhielt Reichstein, vermittelt über den wissenschaftlichen Direktor von Organon Nutley, von Pincus eine entsprechende Anfrage, musste aber ausrichten lassen: ,Unfortunately I do not posses more than a few mgs of 11-dehydrocorticosterone and cannot send you the 0,5 or $1 \mathrm{~g}$ for Dr. Pincus. We are not in a position to prepare these products on a large scale but must confine ore work to find out the methods." StaBS, PA 979a, K 11-2 4: Wissenschaftliche Korrespondenz mit Organon Inc. (vormals Roche-Organon Inc., Nutley), 1940-1949, Brief vom 7. Oktober 1946.

17 Zur Geschichte des Cortisons s. Cantor 1992, Marks and Harry 1992, Rasmussen 2002b, Quirke 2005.

18 Zur Kybernetik s. Pias 2004, Pickering 2007, Hagner 2008.

19 Vgl. das zusammenfassende Abstract zu den Beiträgen des Symposiums über Stress, 16.-18. März 1953, Washington D.C., http://www.dtic.mil/srch/ doc?collection $=\mathrm{t} 3 \& \mathrm{id}=\mathrm{AD} 0615705$ [11.02.2010].

\section{Literatur}

Abderhalden, Emil, 1946. Vitamine, Hormone, Fermente. Ein Buch für Ärzte, Biologen und Studierende. Basel: Benno Schwabe \& Co.

Addison, Thomas, 1855. On the Constitutional and Local Effects of Disease of the Supra-renal Capsules. London: Samuel Highley.

Annals of the Rheumatic Diseases, 1949. The Seventh International Congress on Rheumatic Diseases. Annals of the Rheumatic Diseases, 8, 302-314.

Army Medical Service Graduate School, 1953. Symposium on Stress (16-18 March 1953). Washington D.C.: Walter Reed Army Medical Center.

Bächi, Beat, 2009. Vitamin C für alle! Pharmazeutische Produktion, Vermarktung und Gesundheitspolitik (1933-1953). Zürich: Chronos.

Benison, Saul/Wolfe, Elin L./Barger, A. Clifford, 1987. Walter B. Cannon. The Life and Times of a Young Scientist. Cambridge, Mass.: Harvard University Press.

Binneveld, Hans, 1997. From Shellshock to Combat Stress. A Compoarative History of Military Psychiatry. Amsterdam: Amsterdam University Press.

Birch, Arthur J., 1992. Steroid Hormones and the Luftwaffe. A Venture into Fundamental Strategic Research and some of its Consequences. The Birch Reduction Becomes a Birth Reduction. Steroids, 57, 363-377.

Bomskov, Christian/Bahnsen, Karl, 1935. Biologische Standardisierung des Hormons der Nebennierenrinde. Naunyn-Schmiedeberg's Archives of Pharmacology, 178, $1-14$.

Borck, Cornelius, 2004. Kummer und Sorgen im digitalen Zeitalter. Stress als Erfolgsprodukt der fünfziger Jahre. Archiv für Mediengeschichte, 4, 73-83. 
Brooks, Chandler McC./Koizumi, Kiyomi/Pinkston, James O., Hg., 1975. The Life and Contributions of Walter Bradford Cannon, 1871-1945. His Influence on the Development of Physiology in the Twentieth Century. Papers Delivered at a Centennial Symposium, "The Life and Influence of Walter Bradford Cannon, 1871-1945. The Development of Physiology in this Century", New York, 2--26 May 1972. New York: State University of New York.

Bürgi, Michael/Strasser, Bruno, Hg., 2005. Wissenschaft, Staat, Industrie. Spezialausgabe der Schweizerischen Zeitschrift für Geschichte, 55.

Cannon, Bradford, 1994. Walter Bradford Cannon. Reflections on the Man and his Contributions. International Journal of Stress Management, 1, 145-158.

Cannon, Walter B., 1914. The Emergency Function of the Adrenal Medulla in Pain and the Major Emotions. American Journal of Physiology, 33, 356-372.

Cannon, Walter B., 1915. Bodily Changes in Pain, Hunger, Fear and Rage. An Account of Recent Researches into the Function of Emotional Excitement. New York: D. Appleton and Company.

Cannon, Walter B., 1932. The Wisdom of the Body. New York: W. W. Norton \& Co.

Cantor, David, 1992. Cortisone and the Politics of Drama, 1949-55. In: John V. Pickstone, Hg., Medical Innovations in Historical Perspective. London: Macmillan, 165-184.

Carter, K. Codell, 2003. The Rise of Causal Concepts of Disease. Burlington: Ashgate.

Copeman, William Sydney Charles, 1953. Cortisone and ACTH in Clinical Practice. London: Butterworth \& Co. Ltd.

Copeman, William Sydney Charles, 1964. A Short History of the Gout and the Rheumatic Diseases. Berkeley: University of California Press.

Everse, J. W. R./de Fremery, P., 1932. Measurement of Fatigue in Rats and its Application for Testing Cortical Extracts. Acta Brevia Neerl. Physiol., 2, 152.

Galison, Peter/Hevly, Bruce, Hg., 1992. Big Science. The Growth of Large-scale Research. Stanford, CA: Stanford University Press.

Gaudillière, Jean-Paul, 2004. Hormones, régimes d'innovation et stratégies d'entreprise. Les exemples de Schering et Bayer. Entreprises et histoire, 36, 84-102.

Grandin, Karl/Wormbs, Nina/Widmalm, Sven, Hg., 2004. The Science-Industry Nexus. History, Policy, Implications. Sagamore Beach: Science History Publications.

Gugerli, David/Kupper, Patrick/Speich, Daniel, 2005. Die Zukunftsmaschine. Konjunkturen der ETH Zürich 1855-2005. Zürich: Chronos.

Hagner, Michael, 2008. Vom Aufstieg und Fall der Kybernetik als Universalwissenschaft. In: Michael Hagner und Erich Hörl, Hg., Die Transformation des Humanen. Beiträge zur Kulturgeschichte der Kybernetik. Frankfurt a. M.: Suhrkamp, 38-71.

Haraway, Donna, 1995. Die Biopolitik postmoderner Körper. Konstitutionen des Selbst im Diskurs des Immunsystems. In: Donna Haraway, Hg., Die Neuerfindung der Natur. Primaten, Cyborgs und Frauen. Frankfurt a. M.: Campus, 160-199.

Hartman, Frank A., 1934. Functions of the Adrenal Cortex. Ohio Journal of Science, 34, 4-8.

Hartman, Frank A./MacArthur, C. G./Hartman, W. E., 1927. A Substance which Prolongs the Life of Adrenalectomized Cats. Proceedings of the Society for Experimental Biology and Medicine, 25, 69-79.

Hench, Philipp S., 1938. Effect of Spontaneous Jaundice on Rheumatoid (Atrophic) Arthritis. Attempts to Reproduce the Phenomenon. British Medical Journal, 2, 394-398.

Hench, Philipp S., (1949). The Potential Reversibility of Rheumatoid Arthritis. Proc Staff Meet Mayo Clinic, 24, 167-178.

Hench, Philipp S./Kendall, Edward C./Slocumb, Charles H./Polley, Howard F., 1950. Effects of Cortisone Acetate and Pituitary ACTH on Rheumatoid Arthritis, Rheumatic Fever and Certain Other Condition. Archives of Internal Medicine, 85, 545-666. 
Hoagland, Hudson, 1944. Adventures in the Biological Engineering. Science, New Series, 100, 63-67.

Hobfoll, Stevan E., 1998. Stress, Culture, and Community. The Psychology and Philosophy of Stress. New York: Plenum Press.

Hofer, Hans-Georg, 2006. Von gestressten Körpern und kaputten Autos. Hans Selye (1907-1982) und das "Allgemeine Adaptionssyndrom". Praxis. Schweizerische Rundschau für Medizin, 95, 1347-1350.

Ingle, Dwight J., 1941. Work Performance of Adrenalectomized Rats Treated with 11-Desoxycorticosterone Sodium Phosphate and 11-Desoxy-17-Hydroxycorticosterone. American Journal of Physiology, 133, 676-678.

Jackson, Mark, 2006. In Search of Stability. Hans Selye and the Biology of Stress. Wellcome History, 1-4.

Kaiser, Hanns/Kley, Hans K., 2002. Cortisontherapie. Corticoide in Klinik und Praxis. Stuttgart/New York: Thieme [11. Auflage].

Kendall, Edward C., 1951. Heberden Oration. Cortisone: Questions than Can Be Answered. Annals of the Rheumatic Diseases, 10, 453-456.

Klinkenberg, Norbert, 1987. Cortison. Die Geschichte des Cortisons und der Kortikosteroidtherapie. Ein Beitrag zur Forschungs- und Therapiegeschichte heutiger Medizin. Köln: Pahl-Rugenstein.

Kury, Patrick, 2008. Von der Managerkrankheit zum Stressmanagement. Überlegungen zur Demokratisierung medizinisch-psychologischer Risikofaktoren nach 1945. Referat auf der Internationalen Tagung "Transformationen der Gesundheit zwischen Politik und Kultur - Praktiken der Prävention im europäischen Vergleich (20. Jahrhundert)" am Wissenschaftszentrum Berlin für Sozialforschung (WZB), 24./25. Juli.

Laurence, William L., 1949. Aid in Rheumatoid Arthritis Is Promised by New Hormone. Hormone is Hailed as Aid in Arthritis. The New York Times, April 21, 1949.

Lazarus, Richard S., 1966. Psychological Stress and the Coping Process. New York: McGraw-Hill.

Lazarus, Richard S., 1977. Cognitive and Coping Processes in Emotion. In: Richard S. Lazarus und Alan Monat, Hg., Stress and Coping. An Anthology. New York: Columbia University Press, $145-158$.

Lederer, Susan E., 1992. Political Animals. The Shaping of Biomedical Research Literature in Twentieth-Century America. Isis, 83, 61-79.

Lefrançois, Guy R., 2006. Psychologie des Lernens. Heidelberg: Springer.

Long Hall, Diana, 1976. The Critic and the Advocate. Contrasting British Views on the State of Endocrinolgy in the Early 1920s. Journal of the History of Biology, 9, 269-285.

Marks, Harry M., 1992. Cortisone 1949: A Year in the Political Life of a Drug. Bulletin of the History of Medicine, 66, 419-439.

Nye, Mary Jo, 1996. Before Big Science. The Pursuit of Modern Chemistry and Physics 1800-1940. New York: Twayne Publishers.

Pias, Claus, 2004. Zeit der Kybernetik - eine Einstimmung. In: Claus Pias, Hg., Cybernetics - Kybernetik: The Macy-Conferences 1946-1953. Bd. 2. Zürich/ Berlin: Diaphanes, 9-41.

Pickering, Andrew, 2007. Kybernetik und Neue Ontologien. Berlin: Merve.

Pincus, Gregory/Hoagland, Hudson, 1943. Steroid Excretion and the Stress of Flying. Journal of Aviation Medicine, 14, 173-193.

Pincus, Gregory/Hoagland, Hudson, 1945. Effects on Industrial Production of the Administration of $\Delta 5$ Pregnenolone to Factory Workers, I. Psychosomatic Medicine, 7, 342-346.

Quirke, Viviane, 2005. Making British Cortisone. Glaxo and the Development of Corticosteroid Drugs in the UK in the 1950s and 1960s. Studies in History and Philosophy of Biological and Biomedical Sciences, 36, 645-674.

Rasmussen, Nicholas, 2002a. Of 'Small Men', Big Science and Bigger Business. The Second World War and Biomedical Research in the United States. Minerva, 40, 115-146. 
Rasmussen, Nicholas, 2002b. Steroids in Arms. Science, Government, Industry, and the Hormones of the Adrenal Cortex in the United States, 1930-1950. Medical History, 46, 299-324.

Rasmussen, Nicholas, 2005. The Drug Industry and Clinical Research in Interwar America. Three Types of Physician Collaborator. Bulletin of the History of Medicine, 79, 50-80.

Ratmoko, Christina, 2008. "Geschlecht aus dem Labor". Die Anfänge der industriellen Herstellung von weiblichen und männlichen Hormonen (1914-1939). Dissertation Universität Zürich: Philosophische Fakultät.

Reichstein, Tadeus/Steiger, Marguerite, 1937. Partial Synthesis of a Cristallized Compound with the Biological Activity of the Adrenal-cortical Hormone. Nature, 139, 925-926.

Sarett, Lewis H., 1946. The Structure of some Derivatives of 3( $\alpha)$-Hydroxy- $\Delta 9,11-$ Cholenic Acid. Journal of Biological Chemistry, 162, 591-600.

Sarett, Lewis H./Wallis, Everett S., 1947. The Chemistry of the Steroids. Annual Review of Biochemistry, 16, 655-688.

Schmoch, Ulrich, 2003. Hochschulforschung und Industrieforschung. Perspektiven der Interaktion. Frankfurt a. M.: Campus.

Schönbächler, Georg/Schulthess, Peter, Hg., 2009. Der Emotionsbegriff in den psychotherapeutischen Schulen. Zürich: Collegium Helveticum.

Schwerin, Alexander von, 2009. Prekäre Stoffe. Radiumökonomie, Risikoepisteme und die Etablierung der Radioindikatortechnik in der Zeit des Nationalsozialismus. NTM. Zeitschrift für Geschichte der Wissenschaften, Technik, Medizin, $17,5-33$.

Selye, Hans, 1936. A Syndrome Produced by Diverse Nocuous Agents. Nature, 138, 32.

Selye, Hans, 1944. Hormonal Production of Arthritis. The Journal of the American Medical Association, 124, 201-207.

Selye, Hans, 1946. The General Adaptation Syndrome and the Diseases of Adaption. The Journal of Clinical Endocrinology, 6, 117-230.

Selye, Hans, 1950a. The Physiology and Pathology of Exposure to Stress. A Treatise Based on the Concepts of the General-Adaptation-Syndrome and the Diseases of Adaptation. Montreal: Acta, Inc.

Selye, Hans, 1950b. Stress and the General Adaptation Syndrome. British Medical Journal, 1, 1383-1392.

Selye, Hans, 1952. The Story of the Adaptation Syndrome. Montreal: Acta Inc.

Selye, Hans, 1956. The Stress of Life. London: Longmans, Green and Co.

Selye, Hans, 1975. Homeostasis and the Reactions to Stress. A Discussion of Walter B. Cannon's Contributions. In: Chandler McC. Brooks, Kiyomi Koizumi und James O. Pinkston, Hg., The Life and Contributions of Walter Bradford Cannon, 1871-1945. His Influence on the Development of Physiology in the Twentieth Century. Papers Delivered at a Centennial Symposium Held at State University of New York Downstate Medical Center, 25-26 May 1972. New York: State University of New York Press, 89-107.

Sengoopta, Chandak, 2006. The Most Secret Quintessence of Life. Sex, Glands, and Hormones, 1850-1950. Chicago: The University of Chicago Press.

Siegrist, Johannes, 2001. Work Stress and Health. In: William C. Cockerham, Hg., The Blackwell Companion to Medical Sociology. Oxford: Blackwell, 114-125.

Sinding, Christiane, 1991. Le clinicien et le chercheur. Des maladies de carence à la médicine moléculaire. Paris: Presses Universitaires de France.

Sinding, Christiane, 2001. L'invention de l'insuline, entre physiologie, clinique et industrie pharmaceutique. Médecine sciences, 17, 1176-1181.

Starling, Ernest H., 1923. The Harveian Oration on the Wisdom of the Body. The Lancet, 201, 865-870.

Stoff, Heiko, 2004. Ewige Jugend. Konzepte der Verjüngung vom späten 19. Jahrhundert bis ins Dritte Reich. Köln: Böhlau. 
Stoff, Heiko, 2008. Hormongeschichten. Wie sie in den Jahren 1928 bis 1954 von den Wissenschaftsjournalisten Walter Finkler und Gerhard Venzmer erzählt wurden. Zeitenblicke, 7.

Swingle, Wilbur W., 1927. The Functional Significance of the Suprarenal Cortex. The American Naturalist, 61, 132-146.

Swingle, Wilbur W./Pfiffner, Joseph J./Vars, H. M., 1934. The Cortical Hormone Requirement of the Adrenalectomized Dog, With Special Reference to a Method of Assay. Journal of Biological Chemistry, 104, 701-716.

Tanner, Jakob, 2008. "Fluide Matrix" und "homöostatische Mechanismen". Probleme eines Konzepttransfers zwischen Organismus und Gesellschaft. In: Jörg Martin, Jörg Hardy und Stefan Cartier, Hg., Welt im Fluss. Fallstudien zum Modell der Homöostase. Stuttgart: Franz Steiner Verlag, 11-29.

Uchibayashi, Masao, 2001. Forgotten Episodes of the Birth of Cortisone. Japanese Journal of History of Pharmacy, 36, 70-75.

Viner, Russell, 1999. Putting Stress in Life. Hans Selye and the Making of Stress Theory. Social Studies of Science, 29, 391-410.

Wolfe, Elin L./Barger, A. Clifford/Benison, Saul, 2000. Walter B. Cannon, Science and Society. Boston, Mass.: Boston Medical Library in the Francis A. Countway Library of Medicine.

Wolff, Harold G./Wolf, Stewart/Hare, C. C., Hg., 1950. Life Stress and Bodily Disease. Baltimore: Williams and Wilkins Co.

\section{Lea Haller}

ETH Zürich, Institut für Geschichte/

Technikgeschichte

Auf der Mauer 2 (ADM)

8092 Zürich, Switzerland

E-Mail: haller@history.gess.ethz.ch 